\title{
EOPS: Los corticoides orales, son más o menos eficaces que el budesonide inhalado, para tratar la laringitis subglótica, leve a moderada, en niños?
}

Are oral corticosteroids more or less effective than inhaled budesonide in treating mild to moderate croup in children?

Catalina Brosens*

\begin{abstract}
Resumen
El tratamiento de la laringitis aguda en los niños, sigue siendo muy controvertido. Mientras que algunos sugieren no tratar las laringitis leves, argumentando que se trata de un proceso benigno y autolimitado, otros están a favor de brindar tratamiento farmacológico, ya que éste puede ofrecer beneficios. Tanto el budesonide nebulizado como los corticoides orales, han demostrado mejorar la sintomatología inicial de la laringitis leve a moderada, disminuir el tiempo de estadía en el área de observación de urgencias, disminuir los ingresos hospitalarios y la necesidad de intubación.

La revisión de la literatura mostró que el budesonide y la dexametasona oral, en monodosis, son igualmente eficaces. A la hora de elegir una vía de administración de los corticoides, debe tenerse en cuenta la disponibilidad de la droga, la edad y la colaboración del paciente y de sus padres. Considerando estos parámetros, parecería que la dexametasona oral se convierte en el tratamiento de elección para niños con laringitis leve a moderada, ya que es más económica, está más disponible que el budesonide, y su administración es más sencilla que la nebulización.

Abstract

Croup treatment continues to be controversial. While some doctors suggest not to treat mild croup, since it is a benign and self-limited condition, others favour pharmacologic treatment, because it can offer some benefits. Both nebulized budesonide and oral corticosteroids have demonstrated to improve the symptoms of mild to moderate croup, decrease the length of stay in the emergency department, the hospital admissions and the need of intubation.

The literature review showed that both nebulized budesonide and single dose oral dexamethasone are equally effective. When choosing the route of administration of corticosteroids, we must have in mind the availability of the drug, the age of the patient, and the collaboration that both children and parents can offer. Considering this, oral dexamethasone becomes the best treatment for children with mild to moderate croup, since it is less expensive, it is more available, and is easier to administer than budesonide.
\end{abstract}

Palabras clave: laringitis subglótica, corticoides, niños. Key words: croup, steroids, children.

Brosens C. ¿Los corticoides orales, son más o menos eficaces que el budesonide, para tratar la laringitis subglótica, leve a moderada, en niños? Evid. actual. práct. ambul; 11(5): 157-159, Sep-oct.2008.

\section{Caso clínico}

Consulta a la guardia un niño de 2 años y medio, acompañado por su madre. Son las $10 \mathrm{hs}$ de la mañana. La madre refiere que hace tres días el niño comenzó con un catarro de vías aereas superiores (CVAS) con mocos y algo de tos, sin fiebre, y que en la noche previa se asustó mucho ya que "la tos se intensificó y se convirtió en una tos de perro, la voz se le puso ronca, y parecía como que el aire no le entraba ya que hacía un sonido extraño al respirar". No acudieron a la guardia en ese momento porque el niño se fue tranquilizando cuando la madre lo sacó afuera para que tome aire fresco, pero el episodio asustó mucho a la madre y ella quiere saber si existe algún tratamiento para que esto no vuelva a ocurrir durante la noche, ya que el niño sigue disfónico y con la tos de perro.

Al exámen físico, el niño se encuentra en buen estado general, afebril, con algo de congestión nasal; fauces sin particularidades, con buena entrada de aire bilateral, sin ruidos agregados, con leve tiraje intercostal; la tos es perruna y presenta voz disfónica, con estridor inspiratorio intermitente (sólo presente con el llanto).

Se tranquiliza a la madre diciéndole que su hijo presenta una laringitis subglótica, que es un cuadro viral y autolimitado, y que los corticoides han demostrado ser un tratamiento efectivo para mejorar la sintomatología y disminuir la severidad de la laringitis. En ese momento, me pregunto ¿cuál es la vía de administración más efectiva de los corticoides, oral o inhalatoria, para el tratamiento de la laringitis subglótica leve a moderada, siendo que algunos médicos indican budesonide y otros indican corticoides por vía oral?

\section{Pregunta que generó el caso}

En niños con laringitis subglótica leve a moderada, el tratamiento con budesonide es igual de eficaz que el tratamiento con corticoides administrados por vía oral?

\section{Estrategia de búsqueda bibliográfica}

Se realizó una búsqueda en Medline empleando como palabras clave "laringitis" o "croup" y "tratamiento". Se limitó la búsqueda a estudios controlados aleatorizados y revisiones sistemáticas que tuvieran como máximo 10 años desde su publicación. Se seleccionaron 2 estudios que se describen más adelante.

\section{Laringitis subglótica o viral}

La laringitis subglótica, laringotraqueobronquitis o crup, es un cuadro clínico caracterizado por tos perruna, afonía, estridor inspiratorio y dificultad respiratoria. Es producida casi exclusivamente por agentes virales, el más frecuentemente implicado es el virus parainfluenza. La laringitis subglótica aparece principalmente en niños de entre 3 meses y 3 años, con un pico de incidencia a los 2 años.

La mayoría de los casos se dan en otoño e invierno. Habitualmente es precedido por un CVAS 24 a $72 \mathrm{hs}$ antes, y en forma más o menos brusca, y generalmente por la noche, aparece la tríada típica del crup: tos perruna, afonía y estridor de predominio inspiratorio. El cuadro clínico típico es generalmente de intensidad leve y evolución autolimitada, dura 2 o 3 días, aunque la tos puede persistir por un período mayor.

Escala de severidad del crup de Westley (puntaje máximo de 17):

- Grado de estridor(en reposo, sin llanto): 0:sin estridor; 1: estridor audible con estetoscopio; 2: estridor audible sin el estetoscopio - Grado de retracción costal/subcostal: 0: sin retracción; 1: retracción leve; 2: retracción moderada; 3: retracción severa

- Entrada de aire pulmonar: 0: normal; 1: disminuida; 2: severamente disminuida

- Grado de cianosis: 0: ninguna; 4: cianosis con la agitación; 5: cianosis en reposo

- Nivel de conciencia: 0: normal; 5: alterado

$\mathrm{Si}$ bien la laringitis aguda suele ser un proceso benigno y 
autolimitado, los pacientes pueden beneficiarse con tratamiento farmacológico. Existe un debate continuo sobre las indicaciones, dosis y posología de estos fármacos. El tratamiento estándar incluye:

- humedad ambiental (nebulizaciones con solución fisiológica fría) aunque existe poca evidencia científica de que sea efectiva; - adrenalina nebulizada, para los casos moderados y graves que no altera el curso de la enfermedad, pero produce mejoría clínica, siendo su efecto rápido pero transitorio, de aproximadamente 2 horas;

- corticoides: han demostrado ser efectivos en el manejo ambulatorio de los pacientes con crup leve y moderado, por lo que actualmente son recomendados por todos los expertos.

Inhalados (budesonide nebulizado): para los casos leves y moderados, y de apoyo en los graves, en una dosis fija de $2 \mathrm{mg}$ disuelta en $4 \mathrm{ml}$ de solución fisiológica (independientemente del peso y la edad) que demostró un efecto beneficioso, mejorando la sintomatología inicial, disminuyendo el tiempo de estancia en urgencias, reduciendo la necesidad de adrenalina nebulizada y reduciendo la tasa de hospitalización.

Sistémicos: el más utilizado y estudiado es la dexametasona, ya sea $0,6 \mathrm{mg} / \mathrm{kg}$ por vía intramuscular en dosis única, o dosis inferiores de $0,3 \mathrm{mg} / \mathrm{kg}$ e incluso $0,15 \mathrm{mg} / \mathrm{kg}$ por vía oral en dosis única, en los casos leves, que han probado tener igual eficacia; también disminuyen la gravedad de los síntomas, la necesidad de adrenalina nebulizada, los ingresos hospitalarios, la necesidad de intubación y la estancia en el área de observación de urgencias; si bien existen pocos estudios acerca de la prednisolona o prednisona en el tratamiento de la laringitis, un estudio ha demostrado que dosis equivalentes $(1 \mathrm{mg} / \mathrm{kg})$ de este fármaco administrado en dosis única, es menos efectiva que la dexametasona oral en disminuir posteriores consultas al sistema de salud, sin hallarse diferencias significativas en otros resultados (score del crup, necesidad de adrenalina, tiempo de permanencia en la guardia, duración de los síntomas), sugiriendo que si se sustituye a la dexametasona por prednisolona se utilice una dosis de $1 \mathrm{mg} / \mathrm{kg} /$ día (cada 8 horas) por 2 días, por su menor vida media. Aún no existen estudios que comparen directamente los resultados del tratamiento con monodosis vs. 2 días de tratamiento para corticoides por vía oral.

Frente a pacientes con crup leve, uno podría decidir directamente no tratar, teniendo en cuenta que se trata de un cuadro autolimitado, o indicar únicamente nebulizaciones con solución fisiológica fría, sabiendo que no existe evidencia que avale su uso. Podríamos decidir tratar con monodosis de corticoides (oral o nebulizado) sobre todo a aquellos pacientes con algún factor de riesgo, tal como domicilio lejano, menor de 6 meses, padres poco fiables, etc., o simplemente a aquellos con padres demandantes/ansiosos/agotados.

Existen varios ensayos clínicos en los que se compara el efecto del budesonide nebulizado con la dexametasona oral, concluyendo que son igualmente eficaces.

\section{Resumen de los trabajos encontrados}

Feyzullah Cetinkaya, Bilge Sertel Tüfekci, Günsel Kutluk. A comparison of nebulized budesonide, and intramuscular, and oral dexamethasone for treatment of croup (Una comparación entre el budesonide nebulizado y la dexametasona intramuscular y oral para el tratamiento del crup)
Internacional Journal of Pediatric Otorhinolaryngology 2004; $68,453-456$

Ensayo clínico aleatorizado realizado en el Departamento de Emergencias de Pediatría en el Sisli Eftal Education and Research Hospital en Estambul, Turquía. El estudio compara la eficacia del budesonide nebulizado y de la dexametasona oral e intramuscular, respectivamente, para el tratamiento del crup. Incluyó a 60 niños de entre 6 y 36 meses que se presentaron al departamento de emergencias con el diagnóstico de crup; estos fueron asignados en forma aleatorizada a uno de los 4 grupos (cada uno con 15 niños). El primer grupo recibió 500 ug ( $2 \mathrm{ml}$ ) de budesonide nebulizado + una única dosis de placebo oral $+2 \mathrm{ml}$ de solución fisiológica (placebo) intramuscular; el segundo grupo recibió $2 \mathrm{ml}$ de solución fisiológica nebulizada + una única dosis de placebo oral + una única dosis de 0,6 mg/kg de dexametasona intramuscular; el tercer grupo recibió $2 \mathrm{ml}$ de solución fisiológica nebulizada + una única dosis de $0,6 \mathrm{mg} / \mathrm{kg}$ de dexametasona oral $+2 \mathrm{ml}$ de solución fisiológica intramuscular; el cuarto grupo (grupo placebo) recibió sólo placebo en sus distintas vías de administración. Además todos los grupos recibieron al inicio salbutamol y otras medidas de soporte. Todos los pacientes fueron evaluados con el score de Westley en el momento de admisión al departamento de emergencias ( 0 hs) y a las 24,48 y 72 horas, no existiendo diferencias significativas entre los grupos respecto de la escala de Westley al inicio de la evaluación. Se excluyó a aquellos pacientes que presentaban epiglotitis, aspiración de cuerpo extraño, neumonía aguda bacteriana, anomalías congénitas o adquiridas de la vía aérea superior, inmunodeficiencias, pacientes que habían sido intubados dentro del mes, o que habían recibido corticoides en las 2 semanas previas.

El estudio mostró que los puntajes de la escala de severidad de Westley en los tres grupos de tratamiento a las 24, 48 y 72 horas fueron significativamente más bajos comparados con los puntajes del grupo placebo $(P=0.000)$; y que cuando se comparó a los tres grupos de tratamiento entre sí, no se hallaron diferencias estadísticamente significativas en los puntajes de la escala de severidad del crup $(P>0,05)$. Por lo que concluye que tanto el budesonide nebulizado, como la dexametasona oral e intramuscular, son igualmente eficaces en el tratamiento del crup, y que a la hora de elegir una vía de administración de los corticoides, se deben tener en cuenta la disponibilidad de la droga, la edad y colaboración del paciente y de sus padres.

Terry P. Klassen, MD; William R. Craig, MD, CM; David Moher, MSc; Martin H. Osmond, MD, CM; Hans Pasterkamp, MD; Terry Sutcliffe, BA; Lise K. Watters, MD; Peter C. Rowe, MD. Nebulized Budesonide and Oral Dexamethasone for Treatment of Croup: a Randomized Controlled Trial (Budesonide nebulizado y Dexametasona oral para el Tratamiento del Cruz: un Ensayo Clínico Controlado y Aleatorizado)

JAMA. 1998; 279: 1629-1632

Ensayo clínico controlado y aleatorizado realizado en los departamentos de emergencias de dos hospitales pediátricos de Canadá, entre Octubre de 1995 y Enero de 1997, que incluyó a 198 niños de entre 3 meses y 5 años con cuadro clínico compatible con crup y con un puntaje mayor o igual a 2 de la escala de severidad de Westley. El objetivo del trabajo fue determinar la efectividad de 3 regímenes distintos de corticoides en pacientes con crup leve a moderado. Los pacientes recibieron en forma aleatorizada alguna de las 3 intervenciones: $0,6 \mathrm{mg} / \mathrm{kg}$ de dexametasona oral y placebo nebulizado; placebo oral y $2 \mathrm{mg}$ de budesonide nebulizado; o $0,6 \mathrm{mg} / \mathrm{kg}$ de 
dexametasona oral y $2 \mathrm{mg}$ de budesonide nebulizado. Se midió como evento primario el score de crup de Westley; los resultados secundarios medidos fueron: saturación de $\mathrm{O} 2$, frecuencia cardíaca, y frecuencia respiratoria, los cuales fueron evaluados al momento del ingreso a la guardia, y en forma horaria, hasta llegar a un puntaje de 1 o menor, hasta que el paciente fuera dado de alta por franca mejoría clínica, hasta que el paciente necesitara adrenalina, o hasta que hubieran pasado 4 horas. Otros resultados secundarios que se midieron fueron: la tasa de ingreso hospitalario, el tiempo de permanencia en el departamento de emergencia, visitas posteriores, número de cointervenciones y la persistencia de los síntomas a los 7 días. Se excluyó a aquellos pacientes con epiglotitis; enfermedad pulmonar crónica (excluyendo asma); crup severo (puntaje mayor o igual a 8); pacientes que recibieron tratamiento con adrenalina racémica en la llegada al departamento de emergencia; que recibieron tratamiento con corticoides en las 2 semanas previas; antecedentes de medio de tuberculosis; varicela o exposición a esta dentro de los 21 días previos; o inmunodeficiencia conocida.

La media del puntaje de la escala de severidad de Westley al inicio de la evaluación fue de 3,5 a 3,8. Para el budesonide, se vio que el cambio en la media del puntaje, desde el inicio hasta el final de la evaluación, fue de $-2,3(95 \% \mathrm{Cl},-2,6$ a $-2,0)$; para la dexametasona, $-2,4(95 \% \mathrm{Cl},-2,6$ a $-2,2)$; y para budesonide y dexametasona, $-2,4(95 \% \mathrm{Cl},-2,7$ a $-2,1)$. No se hallaron diferencias significativas entre los tres grupos de tratamiento.
Tampoco se hallaron diferencias significativas en los resultados secundarios.

El estudio concluye que todos los grupos tuvieron un cambio similar en el puntaje de la escala de severidad del crup, en el tiempo de permanencia en la guardia, en el uso de otros tratamientos, y en la persistencia de los síntomas a los 7 días; por lo que no existen diferencias significativas en los resultados clínicos entre los diferentes grupos de tratamiento.

Teniendo en cuenta estos resultados, podría concluirse que la dexametasona por vía oral, en dosis única de 0,15 a $0,6 \mathrm{mg} / \mathrm{kg}$, se convierte en el tratamiento de elección para pacientes con crup leve a moderado, ya que es más económica que el budesonide, está más disponible, y la administración por vía oral es más sencilla que la nebulización, que lleva por lo menos $10 \mathrm{mi}-$ nutos, durante los cuales los niños suelen ofrecer resistencia.

Debemos tener en cuenta que en la Argentina todavía no se dispone de dexametasona en jarabe, ésta esta disponible únicamente está en comprimidos o por vía intramuscular. Una buena opción sería moler el comprimido y mezclarlo con algún alimento blando, sobre todo para los niños más pequeños, para que sea mejor tolerado. Si esta forma de administración de la dexametasona no pudiera llevarse a cabo, debemos saber que una sola dosis de budesonide nebulizado es una alternativa adecuada.

\section{Bibliografía recomendada}

- Bjornson C y col. for the Pediatric Emergency Research Canada Network. A randomized trial of a single dose of oral dexamethasone for mild croup. N Engl J Med 2004;351:1306-13. Cherry J. Croup. N Eng J Med 2008; 358: 384-91.

- Feyzullah C y col. A comparison of nebulized budesonide, and intramuscular, and oral dexamethasone for treatment of croup. International Journal of Pediatric Otorhinolaryngology 2004 68, 453-456.

- Fitzgerald D. Croup: assessment and evidence-based management. MJA 2003; 179 (7): 372-377.

- García Martín F y col. Laringitis. Protocolos diagnósticos y terapéuticos de la A.E.P. 2001; Tomo 2; capitulo 20; $137-146$.

- Luria J y col. Effectiveness of Oral or Nebulizad Dexamethasone for Children UIT Mild Croup. Arch Pediatr Adolesc Med. 2001; 155:1340-1345

- Sparrow A y col. Prednisolone versus dexamethasone in croup: a randomised equivalence trial. Arch. Dis. Child. 2006; 91; 580-583.

- Terry P y col. Nebulized Budesonide and Oral Dexamethasone for Treatment of Croup: a Randomized Controlled Trial. JAMA. 1998; 279: $1629-1632$. 